\title{
Exploring Latent Profiles of Psychopathology in a Sample of Lonely People Seeking Treatment
}

\author{
Anton Käll ${ }^{1}$ (D) $\cdot$ Roz Shafran ${ }^{2}$ (ID $\cdot$ Gerhard Andersson ${ }^{1,3,4}$ (D)
}

Accepted: 26 January 2021 / Published online: 14 February 2021

(C) The Author(s) 2021, corrected publication 2021

\begin{abstract}
Loneliness is a common experience in populations experiencing mental health problems. Associations with symptoms of psychopathology are prevalent, but little is known about the diversity of symptom profiles in people suffering from prolonged and distressing loneliness. The current study sought to investigate the existence of subgroups of loneliness and common forms of psychopathology in a sample of adults $(n=332)$ seeking treatment for their loneliness. Using latent profile analysis, five profiles were identified and differences in demographic factors were investigated. The profiles differed mainly in the severity of symptoms of depression, worry, and social anxiety, with the largest profile exhibiting minimal-to-mild symptoms $(n=109)$ across these indicators. The second largest group was characterized primarily by high ratings of social anxiety $(n=90)$. Overall, three of the five profiles, encompassing roughly half of the sample, scored at a clinical level on mental health symptom measures. Age was inversely related to symptom severity among the profiles, indicating that the older subgroups of the sample exhibited milder symptoms, particularly on social anxiety. The results provide insight into the heterogeneity of the clinical symptoms of people suffering from loneliness and add important knowledge that could help inform how to best intervene to help this population.
\end{abstract}

Keywords Loneliness $\cdot$ Latent profile analysis $\cdot$ Social anxiety $\cdot$ Psychopathology

\section{Introduction}

Loneliness is an aversive emotional state that signals a discrepancy between a person's wanted and actual social situation (Peplau and Perlman 1982). Even if it is an almost ubiquitous experience at times during the life-span, the link between chronic loneliness and adverse somatic and psychological

Anton Käll

anton.kall@liu.se

Roz Shafran

r.shafran@ucl.ac.uk

Gerhard Andersson

gerhard.andersson@liu.se

1 Department of Behavioural Sciences and Learning, Linköping University, SE-581 83 Linköping, Sweden

2 Institute of Child Health, University College London, London WC1N 1EH, UK

3 Department of Biomedical and Clinical Sciences, Linköping University, Linköping, Sweden

4 Department of Clinical Neuroscience, Karolinska Institutet, Stockholm, Sweden consequences is a cause for clinical concern (Heinrich and Gullone 2006; Valtorta et al. 2016). The percentage of the adult population experiencing loneliness 'most of the time' or 'always' has been noted at $6 \%$ in the United Kingdom (Victor and Yang 2012). When focusing specifically on subgroups of the population the percentages often rise substantially, with 11 to $15 \%$ percent of older adults reporting feeling lonely often or always in a cohort study of older adults in Sweden (Dahlberg et al. 2018). In addition to the frequency of loneliness, a study in the general population in Germany indicated that $3.9 \%$ were moderately distressed by their loneliness and that $1.7 \%$ were severely distressed (Beutel et al. 2017).

In the context of psychopathology, loneliness has sometimes been viewed as a symptom of psychiatric disorders. For example, loneliness is listed as a symptom of depression in the Center of Epidemiological Studies - Depression scale (CES-D; Radloff 1977). From a theoretical perspective it has also been suggested that loneliness is a byproduct of an internalizing disposition characterized by high degrees of social anxiety, and thus caused and maintained by the avoidance behaviors seen in social anxiety disorder (Rubin et al. 2009). However, there are reasons to believe that loneliness is a distinct phenomenon as well. Factor analyses have found that loneliness is best represented as separate from both social anxiety and depression 
in samples of young adults (Fung et al. 2017) and adolescents (Danneel et al. 2019a). Cacioppo et al. (2006) reported that loneliness was less characterized by negative affect than expected if loneliness was primarily a symptom of depression.

Bearing this in mind, it is important to note that a relationship between loneliness and symptoms of psychopathology has been found frequently. Loneliness is a frequent complaint among users of mental health services (Wang et al. 2019), and people receiving psychiatric treatment (Lasgaard et al. 2016). In a representative U.K. sample, Meltzer et al. (2013) found loneliness levels to be significantly related to the risk of meeting the criteria for a multitude of psychiatric disorders, including obsessive compulsive disorder, depression, and phobias. The odds ratios for having these diagnoses ranged between 9.66 and 11.66. Other studies have noted substantial correlations with symptoms of depression and generalized anxiety disorder (Beutel et al. 2017), psychosis in a meta-analytic investigation (Michalska da Rocha et al. 2017), and suicidal ideation and attempts whilst controlling for symptoms of common psychiatric disorders (Stickley and Koyanagi 2016). Longitudinal investigations have suggested that a reciprocal link between loneliness and depression (Cacioppo et al. 2010), and with social anxiety (Lim et al. 2016; Maes et al. 2019). A three-wave longitudinal study investigating the relationship between loneliness, symptoms of depression, and symptoms of social anxiety found significant associations between the three constructs, with results suggesting that the link between loneliness and depressive symptoms was influenced through the common connection with social anxiety at earlier timepoints (Danneel et al. 2019b). In sum, though seemingly discrete in nature, loneliness and symptoms of psychopathology are highly interrelated, with several possible pathways linking the constructs.

While the literature suggests that loneliness and symptoms of psychiatric disorders are linked, little is known about the heterogeneity of these symptoms within the population of people suffering from chronic loneliness. There are reasons to suspect that the assumption of 'all is equal' is not an accurate one when considering loneliness. Lasgaard et al. (2011) found that loneliness attributable to lacking quality in connections with family had a close relationship to depressive symptoms, while loneliness due to an unwanted romantic situation was found to covary with social anxiety. On a related note, Timpano et al. (2014) found that symptoms of OCD were most closely related to the aspects of loneliness thought to mirror withdrawal and feelings of isolation, rather than perceived closeness and collective belonging. While these analyses relied on a variable-centered approach, there is also evidence of heterogeneity in studies employing methods specifically designed to detect subgroups. In a latent class analysis using the De Jong-Gierveld Loneliness Scale, Hyland et al. (2019) investigated the specific impact of social loneliness, thought to represent a lack of friends and broader social network, as well as emotional loneliness, thought to represent a lack of closer, more intimate relationships. The results showed that participants reporting high levels of both social and emotional loneliness scored significantly higher on measures of depression and generalized anxiety and lower on a measure of psychological well-being compared to a group who reported mainly social loneliness. While the former subgroup scored higher on measures of psychopathology, the group considered to be primarily emotionally lonely had significantly higher ratings of psychological distress than participants primarily endorsing the items measuring social loneliness. This indicates that different kinds of loneliness are differentially related to symptoms of depression and worry. Using the same measures to assess loneliness, Chiao et al. (2019) reported three clusters in their sample: emotional loners, serious emotional loners, and severe emotional and social loners. The latter two clusters were related to a significantly higher relative risk of depressive symptoms compared to the first cluster. However, the relative risk ratio between those with high levels of emotional loneliness and those with high levels of both emotional and social loneliness was very similar. A third study by Hawkins-Elder et al. (2018) used three items based on the Social Provisions Scale (Cutrona and Russell 1987) to identify latent profiles of loneliness. The results indicated that the subgroup with high levels of loneliness had the highest ratings of psychological distress and neuroticism, while also having low levels of extraversion, in relation to the other subgroups. The findings indicated that the severity of loneliness, rather than kind, is an important factor when identifying the relationship with psychopathology.

In sum, the studies reviewed suggest a heterogeneity within the population of people suffering from loneliness. However, there are some reservations with respect to these findings. No study included a set of instruments measuring both social anxiety and depression, arguably the two constructs most closely related to loneliness (e.g. Danneel et al. 2019a). The results are also inconclusive regarding whether the population is best characterized by linear associations between symptoms (for example scores on measures of depression, social anxiety and loneliness being strongly correlated), or whether there are specific subgroups based on specific symptoms within the population (i.e., one group primarily characterized by symptoms of social anxiety, one primarily by depression etc.). Additionally, while Hyland et al. (2019) used a criterion to distinguish lonely from non-lonely people, they did not focus specifically on the population at the higher end of the loneliness distribution. Understanding heterogeneity and possible subgroups in the loneliness population, in particular in the more severe forms of loneliness and in relation to psychiatric symptoms, could be important for explaining the maintenance and treatment of loneliness over time (Käll et al. 2020b). 
The current study sought to explore the existence of subgroups within a sample of chronically lonely people based on ratings of loneliness and psychopathology. We did this using all available baseline intake data collected in association with two controlled trials of internet-based cognitive behavioral therapy (ICBT) targeting loneliness. Parts of the data have thus been reported in an earlier paper (Käll et al. 2020a). Additionally, we were interested in whether these hypothesized subgroups would differ with regard to demographic characteristics.

\section{Method}

\section{Participants and Procedure}

The participants were recruited as part of a screening procedure for two trials investigating the effects of ICBT targeting chronic loneliness. The intervention targeted adults experiencing frequent and distressing loneliness living in Sweden for whom this experience was a lasting problem. Participants applied to the trials through a secure website where they registered an account and filled in the screening questionnaires (see Vlaescu et al. 2016 for a description of the platform and its functionality). The listed criteria for applying to the studies were a) distress caused by loneliness, b) age 18 years or older, c) ability to read, write, and speak Swedish, d) not currently undergoing any kind of psychological treatment, and e) not having any recent or planned changes in psychotropic medications. The information also specified that we would not be able to include participants with a diagnosed personality disorder, ongoing substance abuse, or a need for acute psychiatric care (e.g., anorexia nervosa, suicidal intent). Other than these criteria, we did not target specific characteristics or demographic groups. Information about the study was spread using equivalent channels in both trials: through advertisements in two national newspapers, social media posts, reports in local newspapers, and posters in cities around Sweden. The materials and information in the media stated that the trials were aimed at potential participants experiencing frequent loneliness. In total, 332 participants applied for participation (of which 243 took part of the treatments). Demographic characteristics can be seen in Table 1 and means for the indicators used in the analysis for the pooled sample can be viewed in Table 2.

\section{Indicators}

\section{UCLA Loneliness Scale, Version 3}

The UCLA Loneliness Scale, version 3 (ULS-3) is 20-item scale meant to capture the experience of loneliness (Russell 1996). The instrument was chosen as it provides a uniform
Table 1 Demographic characteristics of the participants $(n=332)$

\begin{tabular}{|c|c|c|c|c|}
\hline & $n$ & $\%$ & M & SD \\
\hline Age & & & 46.13 & 16.93 \\
\hline \multicolumn{5}{|l|}{ Sex } \\
\hline Female & 247 & 74.4 & & \\
\hline Male & 84 & 25.3 & & \\
\hline Intersex & 1 & 0.3 & & \\
\hline \multicolumn{5}{|l|}{ Civil status } \\
\hline Single & 179 & 53.9 & & \\
\hline Partner/Married & 109 & 32.8 & & \\
\hline Divorced/Widow/Widower & 44 & 13.3 & & \\
\hline \multicolumn{5}{|l|}{ Living with } \\
\hline Alone & 211 & 63.6 & & \\
\hline With family & 60 & 18.1 & & \\
\hline With friends & 7 & 2.1 & & \\
\hline With partner & 44 & 13.3 & & \\
\hline Other communal living & 10 & 3.0 & & \\
\hline \multicolumn{5}{|l|}{ Highest Educational Degree } \\
\hline No completed degree & 2 & 0.6 & & \\
\hline Primary school & 9 & 2.7 & & \\
\hline Secondary school & 82 & 24.7 & & \\
\hline College/University & 195 & 58.7 & & \\
\hline Other vocational education & 33 & 9.9 & & \\
\hline Post-graduate degree & 11 & 3.3 & & \\
\hline \multicolumn{5}{|l|}{ Primary occupation } \\
\hline Student & 47 & 14.2 & & \\
\hline Employed & 171 & 51.5 & & \\
\hline Unemployed & 18 & 5.4 & & \\
\hline Internship & 1 & 0.3 & & \\
\hline Retired & 72 & 21.8 & & \\
\hline Short-term sick leave & 3 & 0.9 & & \\
\hline Long-term sick-leave & 13 & 3.9 & & \\
\hline Other & 7 & 2.1 & & \\
\hline $\begin{array}{l}\text { Previous treatment for mental health } \\
\text { problems: Yes }\end{array}$ & 182 & 54.8 & & \\
\hline $\begin{array}{l}\text { Previous/current use of psychotropic } \\
\text { medication: Yes }\end{array}$ & 155 & 46.7 & & \\
\hline
\end{tabular}

estimate of individual differences in the level of loneliness. Respondents are asked to indicate the frequency of experiences on a four-point scale ranging from "never" (scored as

Table 2 Observed means, standard deviations, and ranges for the measures used in the latent profile analysis

\begin{tabular}{lcrrr}
\hline & $n$ & M & \multicolumn{1}{c}{ SD } & Range \\
\hline UCLA Loneliness Scale, version 3 & 332 & 58.80 & 7.28 & $32-75$ \\
Social Interaction Anxiety Scale & 328 & 35.87 & 17.21 & $0-74$ \\
Patient Health Questionnaire-9 & 328 & 11.48 & 6.39 & $0-27$ \\
Generalized Anxiety Scale-7 & 326 & 7.78 & 5.46 & $0-21$ \\
\hline
\end{tabular}


1) to "always" (scored as 4). An example item is "How often do you feel left out?". Sum scores can range between 20 (indicating minimal loneliness) and 80 (indicating maximum loneliness). The instrument was shown to have a unidimensional structure during the validation of the scale (Russell 1996). Further investigation has indicated that the instrument taps into both aspects of the social/emotional loneliness dichotomy, though primarily capturing the former construct (Cramer and Barry 1999). The scale has shown an excellent internal consistency (Cronbach's $\alpha$ range between .89 and .94 ) and a one-year test-retest reliability of .73 (Russell 1996). The authors reported a mean of $40.08(S D=9.50)$ and 40.14 ( $\mathrm{SD}=9.52)$, in a sample of students and nurses, respectively. A recent national survey of adults in the USA found a weighted estimated mean of 44.03 ( $\mathrm{SE}=0.09$; Bruce et al. 2019). To our knowledge, no norm scores are available for the measure. In the current sample the instrument showed an internal consistency of Cronbach's $\alpha=.86$.

\section{Patient Health Questionnaire-9}

The patient health questionnaire 9-item scale (PHQ-9) is a self-administered instrument assessing the severity of symptoms of major depressive disorder (Kroenke et al. 2001). Respondents are asked to indicate the frequency by which they experience a given symptom on a scale ranging from "Not at all" (scored as 0) to "Nearly every day" (scored as 3 ). The total sum can range from 0 to 27 . Normative severity ranges during validation of the instrument were set to 0 to 4 for minimal, 5 to 9 for mild, 10 to 14 for moderate, 15-19 for moderate severe, and 20-27 for severe depression. The instrument has been reported to have an excellent internal consistency, Cronbach's $\alpha=.89$, and a test-retest reliability of .84 (Kroenke et al. 2001). In the current sample the internal consistency was Cronbach's $\alpha=.86$.

\section{Generalized Anxiety Disorder 7-Item Scale}

The generalized anxiety disorder 7-item scale (GAD-7) is a self-administered instrument used to measure the severity of worry and symptoms linked to generalized anxiety disorder (Spitzer et al. 2006). As with the PHQ-9, respondents are asked to indicate the frequency of symptoms on a scale ranging from "Not at all" (coded as 0 ) to "Nearly every day" (coded as 3), with the total sum ranging from 0 to 21 . Severity levels have been reported as 0 to 4 points (minimal severity), 5-9 points (mild severity), 10-14 (moderate severity), and 15-21 (severe severity). The internal consistency has been reported at Cronbach's $\alpha=0.92$ along with a good testretest reliability, ICC $=0.83$. In the current sample the internal consistency was Cronbach's $\alpha=.90$.

\section{Social Interaction Anxiety Scale}

The social interaction anxiety scale (SIAS) is a selfadministered instrument aimed at capturing severity of anxiety related to social interactions in accordance with the generalized subtype of social phobia from the DSM-III (Mattick and Clarke 1998). The instrument has 20 items and the participants are asked to indicate to which degree a given statement is characteristic of them. Each of the items are scored between 0 (not at all characteristic) and 4 (extremely characteristic), with the total sum ranging from 0 to 80 . Internal consistency has been reported at Cronbach's $\alpha=.94$ along with a 12 -week test-retest reliability of .92. In the initial validation study (Mattick and Clarke 1998), the mean in a sample of patients with a diagnosis of social phobia was found to be 34.6 with a standard deviation of 16.4. In a study examining the factor structure of the instrument, Mörtberg et al. (2017) reported a mean of 44.5 with a standard deviation of 9.6 in a sample diagnosed with social anxiety disorder. For the current study, we refer to scores at or above this mean as clinical levels. The version used in the study was updated to reduce the heteronormativity of an item (item 14), replacing the wording "...of the opposite sex" to "...of the sex/sexes I am interested in". This version of the questionnaire has previously been validated in an internet-administered setting (Lindner et al. 2013). The instrument showed an internal consistency of Cronbach's $\alpha=.94$ in the current sample.

\section{Auxiliary Outcome}

\section{Brunnsviken Brief Quality of Life Inventory}

Brunnsviken Brief Quality of Life Inventory (BBQ) is a 12item scale measuring quality of life (Lindner et al. 2016). The scale consists of six questions assessing the respondents satisfaction with different domains of their life (example item: $I$ am satisfied with how I view my life: I know what means a lot to me, what I believe in, and what I want to do with my life) and six follow-up questions inquiring about the importance of the domain to the respondents quality of life (example item: How I view my life is important for my quality of life). The product of each item pair is then summed up to provide an overall rating of quality of life with a range from 0 to 96 . During validation of the study, the instrument was found to have a Cronbach's $\alpha$ of .76, a test-retest ICC of .86, and a convergent validity with the Quality of Life Inventory (QoLI) of .65 (Lindner et al. 2016). The authors found an optimal cutoff for the distinction between a clinical and non-clinical sample at a sum of 52 during validation of the instrument. Analysis of the current sample indicated an internal consistency of Cronbach's $\alpha=.71$. 


\section{Statistical Analyses}

\section{Estimating Latent Profiles within the Sample}

Identification of subgroups was conducted using a latent profile analysis (LPA) in Mplus version 8.4 (Muthén and Muthén 1998-2019). To allow for interpretation against existing clinical norms and population means, the scores on the questionnaires were analyzed as sum scores. All four of the indicators outlined above were used in the model. Each of the LPA models allowed the estimated means to vary across classes. As a second step in the model estimation two different models were specified. The first model estimated the number of classes with fixed between-class variance for the indicators. The second model allowed for varying variance between the classes. Model fit was evaluated using four fit indices: Log-likelihood, Akaike Information Criterion (AIC), Bayesian Information Criterion (BIC), and Sample-size Adjusted BIC (SSA-BIC). Lower values on these indices indicate a better model fit. In addition to the indices, the model's entropy value was used as well. Entropy is a measure of classification certainty and can range between 0 and 1, with a value closer to the latter indicating greater classification certainty (Wedel and Kamakura 2012). Additionally, two statistical tests were used to guide model selection: the likelihood ratio test (LRT) and the parametric bootstrap likelihood ratio test (BLRT). As per recommendations from simulation studies (Nylund et al. 2007; Tein et al. 2013), priority for the decision on number of classes were given to BIC and the BLRT. Lastly, the derived model needed to be interpretable and possible to relate to the existing theory and previous empirical findings of the field. The number of random starts during the estimation process was set to 1000 . The number of bootstrap draws for the BLRT was set at 200 and the number of iterations for the LRT was set at 25 .

\section{Exploring Demographic Characteristics for each Profile}

While auxiliary variables can be included directly into the LPA models, doing so may alter the class extraction process. Based on the results from a simulation study, Nylund-Gibson and Masyn (2016) recommended that estimation of number of classes should be done as a first step, and that the relationship between class membership and auxiliary variables is analyzed separately. For the present study, we used the Bolck-CroonHagenaars-method (Bolck et al. 2004) available in Mplus 8.4. This method allows for investigations of the effect of class membership on auxiliary variables through the use of the estimated membership probabilities and reduces potential bias by weighting the probabilities relative to the measurement error. A simulation study done by Bakk and Vermunt (2016) indicated that this method produces unbiased estimates under a range of possible data conditions.
For the present analysis, we settled on five auxiliary variables of interest due to their associations with loneliness in their literature and the possibility to meaningfully dichotomize them if needed. The variables were age, sex, quality of life, whether the participant lived alone, and whether the participant attributed their loneliness to a specific event. To allow for dichotomization of the sex variable the one participant who responded with intersex to the question was marked as missing for the analysis of auxiliary variables. For the living alone variable, we re-coded the demographic alternatives as either living alone (coded as 1) or not living alone (all the other alternatives; coded as 0). Data for the item about whether the loneliness was attributed to an event were only available from the second intake, with 231 participants (70\%) providing a response to the question. Estimation was made using maximum likelihood, meaning that the available data were included in the analysis, whether the participants provided a response to all the questionnaires or not. Alpha levels were set at 0.05 .

\section{Results}

Demographic characteristics of the sample are presented in Table 1. Means, standard deviations, and ranges for the indicators used in the latent profile analysis are presented in Table 2.

\section{Latent Profiles}

Fit indices for the estimated models are shown in Table 3 . The choice for the best fitting model was the one consisting of five profiles with variances held equal across profiles. This model had the lowest BIC value and a significantly better fit than the four-profile model as shown by the results from the BLRT. Letting the variances vary across classes did not improve the fit of the model to the data, as indicated by the higher BIC value for the profile estimated using these parameters. Estimated means for the indicators in each profile is presented in Table 4. A graph of the profile means relative to the grand means in the sample is shown in Fig. 1.

Loneliness ratings were high across all profiles with means ranging from $54.02(S E=1.12)$ to $63.22(S E=1.05)$. The first estimated profile was also the one with the largest number of participants in it $(n=109,32.8 \%)$. This profile exhibited below average scores for all the indicators, including mild symptoms of depression and minimal symptoms of worry. We refer to this profile as mild psychopathology. The second profile consisted of 49 participants (14.8\%). This profile, similar to the first profile, exhibited a low average rating of loneliness and social anxiety, but moderate and mild/moderate symptoms of depression and worry, respectively. This group was categorized as moderate depression and worry. The third 
Table 3 Fit indices for the tested models

\begin{tabular}{|c|c|c|c|c|c|c|c|c|}
\hline \multicolumn{9}{|c|}{ Varying means, fixed variances } \\
\hline Number of profiles & Free parameters & LL & AIC & $\mathrm{BIC}$ & A-BIC & Entropy & LRT ( $p$ value) & $\operatorname{BLRT}$ ( $p$ value) \\
\hline 1 profile & 8 & -4616.164 & 9248.327 & 9278.768 & 9253.392 & - & - & - \\
\hline 2 profiles & 13 & -4475.647 & 8977.293 & 9026.760 & 8985.523 & .82 & .0000 & .0000 \\
\hline 3 profiles & 18 & -4440.276 & 8916.552 & 8985.044 & 8927.947 & .70 & .0223 & .0000 \\
\hline 4 profiles & 23 & -4418.526 & 8883.052 & 8970.570 & 8897.613 & .74 & .3298 & .0000 \\
\hline 5 profiles $^{\mathrm{a}}$ & 28 & -4400.312 & 8856.623 & 8963.167 & 8874.350 & .77 & .0789 & .0000 \\
\hline 6 profiles & 33 & -4388.797 & 8843.595 & 8969.164 & 8864.487 & .80 & .0199 & .0100 \\
\hline \multicolumn{9}{|c|}{ Varying means, varying variances } \\
\hline Number of profiles & Parameters & LL & AIC & $\mathrm{BIC}$ & A-BIC & Entropy & LRT & BLRT \\
\hline 5 profiles & 44 & -4359.497 & 8806.994 & 8974.420 & 8834.850 & .77 & .4483 & .0100 \\
\hline
\end{tabular}

Abbreviations: LL Loglikelihood, AIC Akaike Information Criterion, BIC Bayesian Information Criterion, A-BIC Sample-size Adjusted Bayesian Information Criterion, LRT Likelihood Ration Test, BLRT Bootstrapped Likelihood Ratio Test

${ }^{\mathrm{a}}$ Model chosen as best fit to the data

profile was the second largest $(n=90,27.1 \%)$. This profile was characterized by average scores on the PHQ-9 and GAD-7, indicating mild to moderate symptoms of depression and minimal to mild symptoms of worry. However, the scores for social anxiety and loneliness was above average, with the average SIAS score being noted at clinical levels. This profile was named primarily socially anxious. Profile four was the smallest with 22 (6.6\%) participants assigned to it. The average ratings for these participants were high across all indicators, including ratings of social anxiety at a clinical level and severe ratings on the measures of depression and worry. We refer to the profile as severe psychopathology. The fifth profile had the third largest percentage of participants assigned to it $(n=62,18.7 \%)$. This profile exhibited above average scores on all of the indicators. The scores on the PHQ-9 and GAD-7 indicated moderately severe and moderate symptoms of depression and worry, respectively. We refer to this profile as moderate psychopathology.

\section{Exploring Demographic Characteristics for each Profile}

Table 5 shows the relationship between profile membership and the auxiliary variables added to the model using the

Table 4 Estimated means, standard errors, and percentages of the indicators and auxiliary variables in each latent profile

\begin{tabular}{|c|c|c|c|c|c|c|c|c|c|c|}
\hline & \multicolumn{2}{|c|}{$\begin{array}{l}\text { Profile 1: Mild } \\
\text { psychopathology } \\
(n=109)\end{array}$} & \multicolumn{2}{|c|}{$\begin{array}{l}\text { Profile 2: Moderate } \\
\text { depression and worry } \\
(n=49)\end{array}$} & \multicolumn{2}{|c|}{$\begin{array}{l}\text { Profile } 3 \text { : Primarily } \\
\text { socially anxious } \\
(n=90)\end{array}$} & \multicolumn{2}{|c|}{$\begin{array}{l}\text { Profile 4: Severe } \\
\text { psychopathology } \\
(n=22)\end{array}$} & \multicolumn{2}{|c|}{$\begin{array}{l}\text { Profile 5: Moderate } \\
\text { psychopathology }(n=62)\end{array}$} \\
\hline & $M$ & SE & $M$ & SE & $M$ & $\mathrm{SE}$ & $M$ & $\mathrm{SE}$ & $M$ & $\mathrm{SE}$ \\
\hline ULS-3 & 54.02 & 1.12 & 55.41 & 1.05 & 63.22 & 1.05 & 62.47 & 1.73 & 62.70 & 0.96 \\
\hline PHQ-9 & 6.65 & 0.56 & 12.22 & 0.84 & 10.47 & 1.23 & 22.59 & 0.81 & 16.55 & 0.72 \\
\hline SIAS & 23.03 & 1.96 & 25.73 & 2.61 & 46.89 & 3.06 & 44.85 & 4.78 & 48.85 & 3.32 \\
\hline GAD-7 & 3.01 & 0.28 & 9.45 & 0.67 & 5.43 & 0.57 & 19.18 & 0.62 & 13.54 & 0.84 \\
\hline \multicolumn{11}{|l|}{ Auxiliary Variables } \\
\hline Age & 51.94 & 1.78 & 51.11 & 3.15 & 39.78 & 2.06 & 37.61 & 3.66 & 43.75 & 2.63 \\
\hline \multirow[t]{2}{*}{ BBQ } & 39.51 & 2.01 & 34.12 & 2.75 & 24.67 & 1.90 & 26.08 & 3.13 & 22.59 & 2.35 \\
\hline & \multicolumn{2}{|l|}{$\%$ Yes } & \multicolumn{2}{|l|}{$\%$ Yes } & \multicolumn{2}{|l|}{$\%$ Yes } & \multicolumn{2}{|l|}{$\%$ Yes } & \multicolumn{2}{|l|}{$\%$ Yes } \\
\hline Women & \multicolumn{2}{|l|}{73.4} & \multicolumn{2}{|l|}{75.5} & \multicolumn{2}{|l|}{74.4} & \multicolumn{2}{|l|}{68.2} & \multicolumn{2}{|l|}{77.4} \\
\hline Living Alone & \multicolumn{2}{|l|}{68.8} & \multicolumn{2}{|l|}{59.2} & \multicolumn{2}{|l|}{63.3} & \multicolumn{2}{|l|}{59.1} & \multicolumn{2}{|l|}{59.7} \\
\hline Loneliness due to an event $\mathrm{t}^{\mathrm{a}}$ & \multicolumn{2}{|l|}{45.6} & \multicolumn{2}{|l|}{48.4} & \multicolumn{2}{|l|}{31} & \multicolumn{2}{|l|}{30.8} & \multicolumn{2}{|l|}{55.1} \\
\hline
\end{tabular}

Abbreviations: ULS-3 UCLA Loneliness Scale, Version 3, PHQ-9 Patient Health Questionnaire-9, SIAS Social Interaction Anxiety Scale, GAD-7 Generalized Anxiety Disorder 7-item scale, $B B Q$ Brunnsviken Brief Quality of Life Scale

${ }^{a}$ Data available for 231 out of the 332 participants 
Fig. 1 Profile Means for the Indicators Relative to the Estimated Grand Means in the Sample. Abbreviations. GAD$7=$ Generalized Anxiety Disorder 7-item scale; PHQ-9 = Patient Health Questionnaire-9; SIAS = Social Interaction Anxiety Scale; ULS-3 = UCLA Loneliness Scale, Version 3

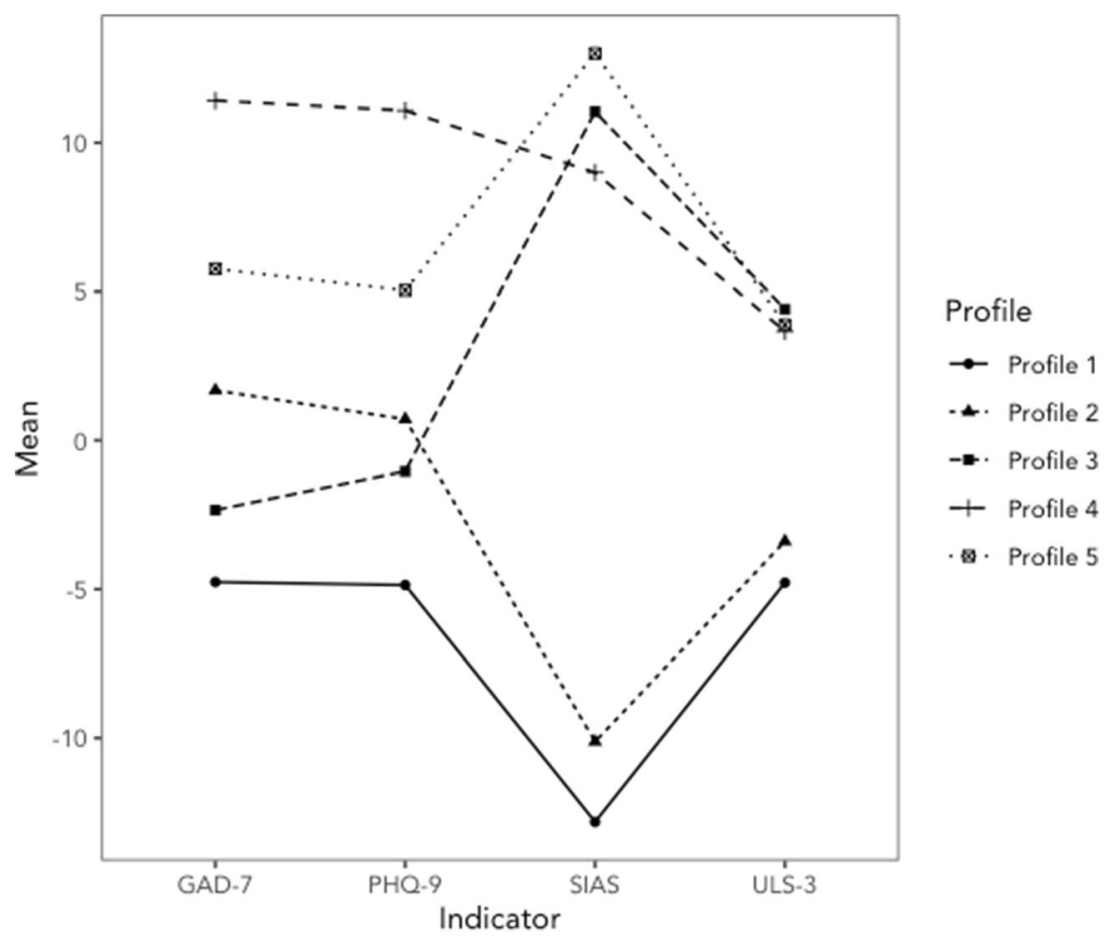

Bolck-Croon-Hagenaars method. The overall test revealed significant differences for two of the four covariates, age and quality of life. When analyzing the pairwise comparisons, the main difference for the age variable was those between profile 1, mild psychopathology, and 2, moderate depression and worry, compared to profile 3, primarily socially anxious, and 4 , severe psychopathology. Additionally, participants in profile 1 were also significantly younger compared to profile 5 , moderate psychopathology. This indicates that participant in the profiles with a higher degree of symptoms of social anxiety (i.e. profile 3, 4, and 5) were younger compared to profile 1 and 2 (except for profile 5) where the symptoms of social anxiety symptoms were less severe.

A similar pattern emerged when analyzing ratings of quality of life. Profile 3, primarily socially anxious, had a significantly lower mean score compared to profile 1, mild psychopathology, and profile 2, moderate depression and worry. The first profile, mild psychopathology, also had significantly higher ratings of quality of life compared to the other profiles, except for profile 2, moderate depression and worry.
Table 5 Overall and pairwise comparisons between estimated latent profiles for the auxiliary variables using the Bolck-CroonHagenaars-Method

\begin{tabular}{lccccc}
\hline & Age & BBQ & $\begin{array}{l}\text { Sex }(\% \\
\text { Women) }\end{array}$ & $\begin{array}{l}\text { Living } \\
\text { alone }\end{array}$ & $\begin{array}{c}\text { Loneliness } \\
\text { attributed } \\
\text { to an event }\end{array}$ \\
\hline Overall Wald $\chi^{2}$ test & $26.651^{* * *}$ & $42.148^{* * *}$ & 0.847 & 2.353 & 8.220 \\
Pairwise comparisons (Wald $\chi^{2}$ test) & & & & \\
Profile 1 vs. Profile 2 & 0.048 & 2.305 & 0.064 & 1.270 & 0.036 \\
Profile 1 vs. Profile 3 & $17.178^{* * *}$ & $24.668^{* * *}$ & 0.003 & 0.526 & 3.643 \\
Profile 1 vs. Profile 4 & $12.364^{* * *}$ & $13.026^{* * *}$ & 0.249 & 0.745 & 1.458 \\
Profile 1 vs. Profile 5 & $6.769^{* *}$ & $30.466^{* * *}$ & 0.407 & 1.212 & 1.089 \\
Profile 2 vs. Profile 3 & $8.068^{* *}$ & $7.124^{* *}$ & 0.073 & 0.204 & 2.615 \\
Profile 2 vs. Profile 4 & $7.886^{* *}$ & 0.401 & 0.401 & 0.005 & 1.401 \\
Profile 2 vs. Profile 5 & 2.824 & 0.070 & 0.070 & 0.018 & 0.310 \\
Profile 3 vs. Profile 4 & 1.321 & 0.446 & 0.192 & 0.104 & 0.022 \\
Profile 3 vs. Profile 5 & 0.267 & 0.149 & 0.355 & 0.115 & $7.086^{*}$ \\
Profile 4 vs. Profile 5 & 1.621 & 0.697 & 0.676 & 0.002 & 2.885 \\
\hline
\end{tabular}

$* p<0.05, * * p<0.01, * * * p<0.001$ 
The omnibus Wald $\chi^{2}$ test did not indicate significant differences between the profiles in terms of the percentage of female participants within the profiles $(p=.932)$, the likelihood of living alone $(p=.671)$, or attributing the feeling of loneliness to a specific event $(p=.084)$.

\section{Discussion}

The aim of this study was to explore the presence of subgroups in a sample of participants applying for treatment of chronic loneliness. Overall, our analyses identified five profiles as the best fit to the data. The loneliness levels within all of these were noted at levels well above the means found in the general population during validation of the instrument used (Russell 1996), and the means found in a more recent representative sample in the U.S. $(\mathrm{M}=44.09, \mathrm{SE}=0.09$; Bruce et al. 2019). These results suggest that we managed to recruit participants with high levels of loneliness. The profiles with clinical levels of social anxiety also had higher levels of loneliness than the profiles with non-clinical levels of social anxiety.

Other than the bimodal distribution of loneliness scores among the profiles, they primarily differed in the severity of symptoms of psychiatric disorders. The estimated profiles suggest that elevated levels of loneliness can be associated with both mild, moderate, and severe symptoms of psychopathology. Most commonly, as with the $32.8 \%$ of the sample classified as mild psychopathology, the indicators suggested a mild severity of depressive symptoms, minimal symptoms of worry and a non-clinical mean score on the social anxiety measure (SIAS). The results from the latent profile analysis indicate that approximately two-thirds of the sample exhibited elevated levels of one or more kinds of psychiatric symptoms, while one-third did not. Given the aforementioned connection between loneliness and symptoms of anxiety disorders and depression (e.g. Meltzer et al. 2013), this was expected. Moreover, the sample used in the analyses represents people with a sufficient level of distress connected to their loneliness to seek help for it, possibly representing a group for which further assessment and treatment could be feasible. Seeing this heterogeneity in symptom severity provides insight into how the mental health profiles of people suffering from distressing and sustained loneliness can be expressed. The results suggest that the relationship between high levels of loneliness and psychiatric disorders might not necessarily be linear, and that there is a diversity to consider when encountering and helping people seeking help for distressing feelings of loneliness.

While the primary factor separating the classes was the severity of psychiatric symptoms overall, participants in the second largest profile, Profile 3, were characterized primarily by social anxiety. This, along with the fact that more than half of the sample exhibited clinical levels of social anxiety, indicates that inquiring about social anxiety and related symptoms is an important part of assessing and understanding clinically relevant loneliness. The overlap is understandable from a theoretical point of view. The maladaptive cognitive and perceptual processes linked to loneliness (for an overview, see Cacioppo and Hawkley 2009), are often found among populations with high levels of social anxiety as well, such as for example difficulties disengaging from threatening social stimuli (Buckner et al. 2010). Maladaptive behavioral patterns, such as avoidance, have also been hypothesized to be of relevance for both social anxiety (Piccirillo et al. 2016) and loneliness (Nurmi et al. 1997). It has been proposed that a modular view of how loneliness develops and is maintained might be the best fit to the available literature (Käll et al. $2020 \mathrm{~b}$ ), which is in line with the findings in the current study. The modular theory suggests that there are different pathways that maintain chronic loneliness over time. In some cases, such as for those with comorbid social anxiety, the maintenance may in large part depend on maladaptive cognitive and behavioral tendencies. Others may struggle mostly due to lacking social skills. The results of the latent profile analysis suggest the existence subgroups with differences both in severity and types of psychiatric symptoms. The results also indicate that a clinical level of social anxiety is a common, but not essential, characteristic of people seeking help for loneliness. Because of this, the assessment of social anxiety and related dysfunctional behavioral and cognitive processes is important when assessing loneliness in a clinical setting. Additionally, future studies could investigate whether subgroups with high levels of social anxiety and loneliness require and/or benefit from a specific focus on these symptoms through, for example, use of behavioral experiments (McManus et al. 2009) and exposure techniques (Hope et al. 2019).

The existence of elevated symptoms levels of one or more kinds of psychopathology in a majority of the sample calls into question about how to best help this population. Should priority be given to the feelings of loneliness and the factors thought to perpetuate these feelings, or is the best way to address symptoms of psychiatric disorders in order to reduce the loneliness? VanderWeele et al. (2011) conducted a marginal structural model analysis concluding that interventions addressing loneliness should also help reduce symptoms of depression. Furthermore, when analyzing the secondary outcomes in a depression trial, Soucy et al. (2019) did not find any significant benefits from neither physical activity nor behavioral activation in reducing loneliness compared to the control group, although the loneliness levels did decrease in the sample overall. For social anxiety, data from a trial of cognitive behavioral therapy (CBT) for children and adolescents have shown decreasing levels of loneliness to mediate decreases in symptoms of social anxiety (Alfano et al. 2009). Results from a later study found that a persisting diagnosis of social anxiety disorder after the conclusion of a CBT treatment was related to higher levels of 
loneliness among the adolescents participating in the trial (Suveg et al. 2017). Based on these results, giving priority to assessing and alleviating the loneliness itself could be the best way in cases where the problems coexist, though researchers should seek to determine this empirically. Additionally, future studies should seek to clarify whether the presence of psychiatric symptoms is of relevance for the outcome of interventions targeting loneliness.

Overall, the profiles differed significantly on two of the five auxiliary variables included in the final model, age and quality of life. The subgroups with higher ratings of social anxiety were also significantly younger on average than participants in the profiles with non-clinical levels of social anxiety. This finding may be an additional indication of the importance of assessing social anxiety in younger people expressing concern about their loneliness. However, it should be noted that the mean ages for the profiles in question (profiles 3 to 5) were around 40 years old with rather large standard errors. Due to this, there would seem to exist substantial heterogeneity within the profiles as well, and the association would seem to not strictly be limited to younger adults. For quality of life, all of the profile means fell well short of the clinical cut-off (Lindner et al. 2016), suggesting an overall low quality of life in all the subgroups. For the comparisons between the profiles, an inverse relationship was found in relation to age. Profiles 1, Mild psychopathology, and 2, Moderate depression and worry, reported significantly higher ratings of quality of live compared to the three other profiles. For the other three auxiliary variables included in the model, none showed significant differences between the profiles.

While the current study provides novel results from a population where the literature is still sparse, there are limitations to consider. First, the sample was rather small, and due to this the final model could not be validated by randomly splitting the data into two samples and confirming the model fit independently in a subset of the larger sample. Moreover, the study was exploratory, and the results should be viewed as tentative until the model fit has been evaluated in other, larger samples. It remains to be seen whether the findings can be generalized across countries and cultural contexts.

Second, ideally the current analyses would have incorporated the change in loneliness after the intervention as a way of analyzing whether profile membership was related to the outcome at posttreatment. The reasoning behind the decision not to do this was twofold: first, outcome data were not available for all participants as some were not included in the treatment studies for which parts of the current data served as the pre-treatment assessment. The main reason for this was that the participants did not complete the structured telephone interview that served as part of the inclusion process for the treatment studies. Second, the interventions which the participants took part in differed both in kind (internet-based interpersonal psychotherapy and internet-based cognitive behavioral therapy) and content (eight weeks for the pilot trial, nine weeks for the follow-up with changes to the order of the material). While the present study did not investigate the predictive properties in terms of the response to psychological treatments or other kinds of interventions, future studies could focus on the role of pretreatment characteristics and subgroups in relation to treatment response.

Third, although the sample was recruited from the general population, the exclusion criteria that was used for the studies could have served to exclude groups where loneliness is a common occurrence. For example, while psychotic symptoms have been linked to loneliness (Michalska da Rocha et al. 2017), these symptoms were listed as a reason for exclusion on the website. Although the aim was to recruit as representative a sample as possible of participants experiencing chronic loneliness, the few exclusion criteria that were employed should be kept in mind when trying to generalize our findings, especially to psychiatric populations.

In summary, the current study identified significant heterogeneity in a sample of participants experiencing loneliness, with a five subgroup-model providing the best fit to the data. The profiles mainly differed in symptom severity on the measures of depression, worry and social anxiety, but the second largest subgroup was characterized mainly by their clinical levels of social anxiety. The results suggest that severe loneliness can exist with or without the presence of symptoms of common forms of psychopathology, but that clinical levels of these symptoms are common. Age could be an important variable of interest; the profiles with an older mean age were characterized by lower overall severity ratings for the indicators in general, and more specifically by their non-clinical ratings of social anxiety. Future studies should aim to replicate these findings and investigate whether individuals within the different profiles benefit from interventions incorporating a specific focus on the comorbid symptoms of psychiatric disorders, such as depression or social anxiety.

Acknowledgements All research at Great Ormond Street Hospital NHS Foundation Trust and UCL Great Ormond Street Institute of Child Health is made possible by the NIHR Great Ormond Street Hospital Biomedical Research Centre. The views expressed are those of the author(s) and not necessarily those of the NHS, the NIHR or the Department of Health.

Funding Open Access funding provided by Linköping University. This study was sponsored in part by a professor grant by Linköping University to the last author.

Data/Material Availability Not available due to the fact that participants were not informed of possibility of data-sharing before consenting to participation in the first trial.

Code Availability Available on request.

\section{Declarations}

Conflict of Interest Anton Käll, Roz Shafran and Gerhard Andersson report no potential conflicts of interest. 
Experiment Participants The study was approved by the Regional Ethics Board (REB). All participants provided informed consent as part of the registration process.

Ethical Approval Ethical approval for the study was provided by the regional ethics board (Identifier: 2015/418-31). All procedures performed in studies involving human participants were in accordance with the ethical standards of the institutional and/or national research committee and with the 1964 Helsinki declaration and its later amendments or comparable ethical standards.

\section{Informed Consent All participants provided informed consent.}

Open Access This article is licensed under a Creative Commons Attribution 4.0 International License, which permits use, sharing, adaptation, distribution and reproduction in any medium or format, as long as you give appropriate credit to the original author(s) and the source, provide a link to the Creative Commons licence, and indicate if changes were made. The images or other third party material in this article are included in the article's Creative Commons licence, unless indicated otherwise in a credit line to the material. If material is not included in the article's Creative Commons licence and your intended use is not permitted by statutory regulation or exceeds the permitted use, you will need to obtain permission directly from the copyright holder. To view a copy of this licence, visit http://creativecommons.org/licenses/by/4.0/.

\section{References}

Alfano, C. A., Pina, A. A., Villalta, I. K., Beidel, D. C., Ammerman, R. T., \& Crosby, L. E. (2009). Mediators and moderators of outcome in the behavioral treatment of childhood social phobia. Journal of the American Academy of Child \& Adolescent Psychiatry, 48, 945-953. https://doi.org/10.1097/CHI.0b013e3181af8216.

Bakk, Z., \& Vermunt, J. K. (2016). Robustness of stepwise latent class modeling with continuous distal outcomes. Structural Equation Modeling: A Multidisciplinary Journal, 23, 20-31. https://doi.org/ 10.1080/10705511.2014.955104.

Beutel, M. E., Klein, E. M., Brähler, E., Reiner, I., Jünger, C., Michal, M., Wiltink, J., Wild, P. S., Münzel, T., Lackner, K. J., \& Tibubos, A. N. (2017). Loneliness in the general population: Prevalence, determinants and relations to mental health. BMC Psychiatry, 17, 1-7. https://doi.org/10.1186/s12888-017-1262-x.

Bolck, A., Croon, M., \& Hagenaars, J. (2004). Estimating latent structure models with categorical variables: One-step versus three-step estimators. Political Analysis, 12, 3-27. https://doi.org/10.1093/pan/ mph001.

Bruce, L. D., Wu, J. S., Lustig, S. L., Russell, D. W., \& Nemecek, D. A. (2019). Loneliness in the United States: A 2018 national panel survey of demographic, structural, cognitive, and behavioral characteristics. American Journal of Health Promotion, 33, 1123-1133. https://doi.org/10.1177/0890117119856551.

Buckner, J. D., Maner, J. K., \& Schmidt, N. B. (2010). Difficulty disengaging attention from social threat in social anxiety. Cognitive Therapy and Research, 34, 99-105. https://doi.org/10. 1007/s10608-008-9205-y.

Cacioppo, J. T., \& Hawkley, L. C. (2009). Perceived social isolation and cognition. Trends in Cognitive Sciences, 13, 447-454. https://doi. org/10.1016/j.tics.2009.06.005.

Cacioppo, J. T., Hawkley, L. C., Ernst, J. M., Burleson, M., Berntson, G. G., Nouriani, B., \& Spiegel, D. (2006). Loneliness within a nomological net: An evolutionary perspective. Journal of Research in Personality, 40, 1054-1085. https://doi.org/10.1016/j.jp.2005.11.007.
Cacioppo, J. T., Hawkley, L. C., \& Thisted, R. A. (2010). Perceived social isolation makes me sad: 5-year cross-lagged analyses of loneliness and depressive symptomatology in the Chicago health, aging, and social relations study. Psychology and Aging, 25, 453-463. https://doi.org/10.1037/a0017216.

Chiao, C., Chen, Y.-H., \& Yi, C.-C. (2019). Loneliness in young adulthood: Its intersecting forms and its association with psychological well-being and family characteristics in northern Taiwan. PLoS One, 14, e0217777. https://doi.org/10.1371/journal.pone.0217777.

Cramer, K. M., \& Barry, J. E. (1999). Conceptualizations and measures of loneliness: A comparison of subscales. Personality and Individual Differences, 27, 491-502. https://doi.org/10.1016/ S0191-8869(98)00257-8.

Cutrona, C. E., \& Russell, D. W. (1987). The provisions of social relationships and adaptation to stress. In W. H. Jones \& D. Perlman (Eds.), Advances in personal relationships (Vol. 1, pp. 37-67). Greenwich: JAI Press.

Dahlberg, L., Agahi, N., \& Lennartsson, C. (2018). Lonelier than ever? Loneliness of older people over two decades. Archives of Gerontology and Geriatrics, 75, 96-103. https://doi.org/10.1016/j. archger.2017.11.004.

Danneel, S., Bijttebier, P., Bastin, M., Colpin, H., van den Noortgate, W., van Leeuwen, K., Verschueren, K., \& Goossens, L. (2019a). Loneliness, social anxiety, and depressive symptoms in adolescence: Examining their distinctiveness through factor analysis. Journal of Child and Family Studies, 28, 1326-1336. https://doi. org/10.1007/s10826-019-01354-3.

Danneel, S., Nelemans, S., Spithoven, A., Bastin, M., Bijttebier, P., Colpin, H., van den Noortgate, W., van Leeuwen, K., Verschueren, K., \& Goossens, L. (2019b). Internalizing problems in adolescence: Linking loneliness, social anxiety symptoms, and depressive symptoms over time. Journal of Abnormal Child Psychology, 47, 16911705. https://doi.org/10.1007/s10802-019-00539-0.

Fung, K., Paterson, D., \& Alden, L. E. (2017). Are social anxiety and loneliness best conceptualized as a unitary trait? Journal of Social \& Clinical Psychology, 36, 335-345. https://doi.org/10.1521/jscp. 2017.36.4.335.

Hawkins-Elder, H., Milfont, T. L., Hammond, M. D., \& Sibley, C. G. (2018). Who are the lonely? A typology of loneliness in New Zealand. Australian \& New Zealand Journal of Psychiatry, 52, 357-364. https://doi.org/10.1177/0004867417718944.

Heinrich, L. M., \& Gullone, E. (2006). The clinical significance of loneliness: A literature review. Clinical Psychology Review, 26, 695718. https://doi.org/10.1016/j.cpr.2006.04.002.

Hope, D. A., Heimberg, R. G., \& Turk, C. L. (2019). Managing social anxiety: A cognitive-behavioral therapy approach: Therapist guide. New York: Oxford University Press.

Hyland, P., Shevlin, M., Cloitre, M., Karatzias, T., Vallières, F., McGinty, G., Fox, R., \& Power, J. M. (2019). Quality not quantity: Loneliness subtypes, psychological trauma, and mental health in the US adult population. Social Psychiatry and Psychiatric Epidemiology, 54, 1089-1099. https://doi.org/10.1007/s00127-018-1597-8.

Käll, A., Jägholm, S., Hesser, H., Andersson, F., Mathaldi, A., Norkvist, B. T., Shafran, R., \& Andersson, G. (2020a). Internet-based cognitive behavior therapy for loneliness: A pilot randomized controlled trial. Behavior Therapy, 51, 54-68. https://doi.org/10.1016/j.beth. 2019.05.001.

Käll, A., Shafran, R., Lindegaard, T., Bennett, S., Cooper, Z., Coughtrey, A., \& Andersson, G. (2020b). A common elements approach to the development of a modular cognitive behavioral theory for chronic loneliness. Journal of Consulting and Clinical Psychology, 88, 269282. https://doi.org/10.1037/ccp0000454.

Kroenke, K., Spitzer, R. L., \& Williams, J. B. W. (2001). The PHQ-9: Validity of a brief depression severity measure. Journal of General Internal Medicine, 16, 606-613. https://doi.org/10.1046/j.15251497.2001.016009606.x. 
Lasgaard, M., Goossens, L., Bramsen, R. H., Trillingsgaard, T., \& Elklit, A. (2011). Different sources of loneliness are associated with different forms of psychopathology in adolescence. Journal of Research in Personality, 45, 233-237. https://doi.org/10.1016/j.jrp.2010.12.005.

Lasgaard, M., Friis, K., \& Shevlin, M. (2016). "Where are all the lonely people?" a population-based study of high-risk groups across the life span. Social Psychiatry and Psychiatric Epidemiology, 51, 13731384. https://doi.org/10.1007/s00127-016-1279-3.

Lim, M. H., Rodebaugh, T. L., Zyphur, M. J., \& Gleeson, J. F. M. (2016). Loneliness over time: The crucial role of social anxiety. Journal of Abnormal Psychology, 125, 620-630. https://doi.org/10.1037/ abn0000162.

Lindner, P., Martell, C., Bergström, J., Andersson, G., \& Carlbring, P. (2013). Clinical validation of a non-heteronormative version of the social interaction anxiety scale (SIAS). Health and Quality of Life Outcomes, 11, 209. https://doi.org/10.1186/1477-7525-11-209.

Lindner, P., Frykheden, O., Forsström, D., Andersson, E., Ljótsson, B., Hedman, E., Andersson, G., \& Carlbring, P. (2016). The Brunnsviken brief quality of life scale (BBQ): Development and psychometric evaluation. Cognitive Behaviour Therapy, 45, 182 195. https://doi.org/10.1080/16506073.2016.1143526.

Maes, M., Nelemans, S. A., Danneel, S., Fernández-Castilla, B., Van den Noortgate, W., Goossens, L., \& Vanhalst, J. (2019). Loneliness and social anxiety across childhood and adolescence: Multilevel meta-analyses of cross-sectional and longitudinal associations. Developmental Psychology, 55, 1548-1565. https://doi.org/10.1037/dev0000719.

Mattick, R. P., \& Clarke, J. C. (1998). Development and validation of measures of social phobia scrutiny fear and social interaction anxiety. Behaviour Research and Therapy, 36, 455-470. https://doi.org/ 10.1016/S0005-7967(97)10031-6.

McManus, F., Clark, D. M., Grey, N., Wild, J., Hirsch, C., Fennell, M., Hackmann, A., Waddington, L., Liness, S., \& Manley, J. (2009). A demonstration of the efficacy of two of the components of cognitive therapy for social phobia. Journal of Anxiety Disorders, 23, 496503. https://doi.org/10.1016/j.janxdis.2008.10.010.

Meltzer, H., Bebbington, P., Dennis, M., Jenkins, R., McManus, S., \& Brugha, T. (2013). Feelings of loneliness among adults with mental disorder. Social Psychiatry \& Psychiatric Epidemiology, 48, 5-13. https://doi.org/10.1007/s00127-012-0515-8.

Michalska da Rocha, B., Rhodes, S., Vasilopoulou, E., \& Hutton, P. (2017). Loneliness in psychosis: A meta-analytical review. Schizophrenia Bulletin, 44, 114-125. https://doi.org/10.1093/schbul/sbx036.

Mörtberg, E., Reuterskiöld, L., Tillfors, M., Furmark, T., \& Öst, L.-G. (2017). Factor solutions of the social phobia scale (SPS) and the social interaction anxiety scale (SIAS) in a Swedish population. Cognitive Behaviour Therapy, 46, 300-314. https://doi.org/10. 1080/16506073.2016.1258723.

Muthén, B. O., \& Muthén, L. K. (1998-2019). Mplus user's guide. Los Angeles: Muthén \& Muthén.

Nurmi, J. E., Toivonen, S., Salmela-Aro, K., \& Eronen, S. (1997). Social strategies and loneliness. The Journal of Social Psychology, 137, 764-777. https://doi.org/10.1080/00224549709595497.

Nylund, K. L., Asparouhov, T., \& Muthén, B. O. (2007). Deciding on the number of classes in latent class analysis and growth mixture modeling: A Monte Carlo simulation study. Structural Equation Modeling: A Multidisciplinary Journal, 14, 535-569. https://doi. org/10.1080/10705510701575396.

Nylund-Gibson, K., \& Masyn, K. E. (2016). Covariates and mixture modeling: Results of a simulation study exploring the impact of misspecified effects on class enumeration. Structural Equation Modeling: A Multidisciplinary Journal, 23, 782-797. https://doi. org/10.1080/10705511.2016.1221313.

Peplau, L. A., \& Perlman, D. (1982). Loneliness: A sourcebook of current theory, research and therapy. New York: Wiley.

Piccirillo, M. L., Taylor Dryman, M., \& Heimberg, R. G. (2016). Safety behaviors in adults with social anxiety: Review and future directions. Behavior Therapy, 47, 675-687. https://doi.org/10. 1016/j.beth.2015.11.005.

Radloff, L. S. (1977). The CES-D scale: A self-report depression scale for research in the general population. Applied Psychological Measurement, 1, 385-401. https://doi.org/10.1177/014662167700100306.

Rubin, K. H., Coplan, R. J., \& Bowker, J. C. (2009). Social withdrawal in childhood. Annual Review of Psychology, 60, 141-171. https://doi. org/10.1146/annurev.psych.60.110707.163642.

Russell, D. W. (1996). UCLA loneliness scale (version 3): Reliability, validity, and factor structure. Journal of Personality Assessment, 66, 20-40. https://doi.org/10.1207/s15327752jpa6601_2.

Soucy, I., Provencher, M. D., Fortier, M., \& McFadden, T. (2019). Secondary outcomes of the guided self-help behavioral activation and physical activity for depression trial. Journal of Mental Health, 28, 410-418. https://doi.org/10.1080/09638237.2018.1466031.

Spitzer, R. L., Williams, J. B. W., Kroenke, K., \& Löwe, B. (2006). A brief measure for assessing generalized anxiety disorder: The GAD7. Archives of Internal Medicine, 166, 1092-1097. https://doi.org/ 10.1001/archinte.166.10.1092.

Stickley, A., \& Koyanagi, A. (2016). Loneliness, common mental disorders and suicidal behavior: Findings from a general population survey. Journal of Affective Disorders, 197, 81-87. https://doi.org/10. 1016/j.jad.2016.02.054.

Suveg, C., Kingery, J. N., Davis, M., Jones, A., Whitehead, M., \& Jacob, M. L. (2017). Still lonely: Social adjustment of youth with and without social anxiety disorder following cognitive behavioral therapy. Journal of Anxiety Disorders, 52, 72-78. https://doi.org/10. 1016/j.janxdis.2017.10.005.

Tein, J.-Y., Coxe, S., \& Cham, H. (2013). Statistical power to detect the correct number of classes in latent profile analysis. Structural Equation Modeling: A Multidisciplinary Journal, 20, 640-657. https://doi.org/10.1080/10705511.2013.824781.

Timpano, K. R., Çek, D., Rubenstein, L. M., Murphy, D., \& Schmidt, N. B. (2014). Exploring the association between obsessive-compulsive symptoms and loneliness: Consideration of specificity and gender. Journal of Cognitive Psychotherapy, 28, 264-273. https://doi.org/ 10.1891/0889-8391.28.4.264

Valtorta, N. K., Kanaan, M., Gilbody, S., Ronzi, S., \& Hanratty, B. (2016). Loneliness and social isolation as risk factors for coronary heart disease and stroke: Systematic review and meta-analysis of longitudinal observational studies. Heart, 102, 1009-1016. https:// doi.org/10.1136/heartjnl-2015-308790.

VanderWeele, T. J., Hawkley, L. C., Thisted, R. A., \& Cacioppo, J. T. (2011). A marginal structural model analysis for loneliness: Implications for intervention trials and clinical practice. Journal of Consulting and Clinical Psychology, 79, 225-235. https://doi.org/ 10.1037/a0022610.

Victor, C. R., \& Yang, K. (2012). The prevalence of loneliness among adults: A case study of the United Kingdom. The Journal of Psychology, 146, 85-104. https://doi.org/10.1080/00223980.2011.613875.

Vlaescu, G., Alasjö, A., Miloff, A., Carlbring, P., \& Andersson, G. (2016). Features and functionality of the Iterapi platform for internet-based psychological treatment. Internet Interventions, 6 , 107-114. https://doi.org/10.1016/j.invent.2016.09.006.

Wang, J., Lloyd-Evans, B., Marston, L., Ma, R., Mann, F., Solmi, F., \& Johnson, S. (2019). Epidemiology of loneliness in a cohort of UK mental health community crisis service users. Social Psychiatry and Psychiatric Epidemiology., 55, 811-822. https://doi.org/10.1007/ s00127-019-01734-6.

Wedel, M., \& Kamakura, W. A. (2012). Market segmentation: Conceptual and methodological foundations (Vol. 8). New York: Springer Science \& Business Media.

Publisher's Note Springer Nature remains neutral with regard to jurisdictional claims in published maps and institutional affiliations. 